\title{
SCSBE: Secured Cluster and Sleep Based Energy-Efficient Sensory Data Collection with Mobile Sinks
}

\author{
S. Balaji1 ${ }^{1}$ Y. Harold Robinson ${ }^{1 *}$, M. Rajaram² \\ ${ }^{1}$ Department of Computer Science and Engineering, SCAD College of Engineering and Technology, \\ Cheranmahadevi, India \\ ${ }^{2}$ Vice-Chancellor, Anna University, Chennai, India \\ Email: sbalajiphd@gmail.com, “yhrobinphd@gmail.com, rajaramgct@rediffmail.com
}

Received 20 April 2016; accepted 15 May 2016; published 28 June 2016

Copyright (C) 2016 by authors and Scientific Research Publishing Inc.

This work is licensed under the Creative Commons Attribution International License (CC BY). http://creativecommons.org/licenses/by/4.0/

(c) (i) Open Access

\begin{abstract}
Wireless sensor networks applications involve a position of inaccessible metropolitan vicinity enclosed by wireless sensor nodes (WSNs)-monitors environmental parameters like battle field surveillance, home applications like fire alarm, health monitoring, etc. Energy plays a vital role in Wireless sensor networks. So, we have to concentrate more on balanced energy consumption for maximizing the network lifetime. Minimizing the whole network overhead and vigor disbursement coupled with the multi-hop data reclamation process that ensuring balanced energy consumption among SNs which results in prolonged network lifetime. This can be achieved by forwarding the sensed data to their cluster heads and then filtering the data before sending it to their tryst nodes, which is located in proximity to MS's trajectory. Sleep and awakening of nodes periodically helps to retain their energy for some more time. The events occurring in any part of the network should be identified by the nodes, while arrangements sleep and active among the nodes. (i.e.) the nodes should be scheduled to sleep, so that the outstanding nodes can take care of the whole network. The eXtensible Randomized Matrix Arithmetic Coding (XRMAC) Technique has been used to enhance the security among all the nodes in the network. Simulation results show that our Proposed Scheme can have better Lifetime, improved throughput, reduced delay compared to other existing methods.
\end{abstract}

\section{Keywords}

Mobile Sinks, Wireless Sensor Networks, Clustering, Tryst Nodes, Sleep and Active

\footnotetext{
"Corresponding author.
}

How to cite this paper: Balaji, S., Harold Robinson, Y. and Rajaram, M. (2016) SCSBE: Secured Cluster and Sleep Based Energy-Efficient Sensory Data Collection with Mobile Sinks. Circuits and Systems, 7, 1992-2001. 


\section{Introduction}

Wireless Sensor Network (WSN) is a collection of sensors with limited resources that collaborate in order to achieve a common goal [1]. Sensor networks are spatially distributed sensors to monitor conditions at different locations, such as strain, hotness, reverberation, shuddering, gesture or pollutants. These tiny sensor nodes, which consist of intellection, data dispensation, and communicating mechanism, pull the idea of sensor networks based on mutual effort of a large number of nodes [2]. A WSN can be deployed in harsh environments to fulfill both military and civil applications. Factors influencing sensor network design are Fault tolerance, Scalability, Operating environment, Sensor network topology, Transmission media and Power consumption [3].

A main reason of energy spending in WSNs relates with communicating the sensor readings from the sensor nodes (SNs) to remote sinks [4]. Energy is very critical resource in wireless sensor networks and can't be recharged frequently. So, we have to think more and more about minimizing the energy consumption of sensor nodes [5]. Nodes - more rapidly to this descend are heavily used to spread data from all network nodes and so, their energy is consumed faster, leading to a non-uniform depletion of energy in the WSN. This results in network disconnections and limited network lifetime [6].

XRMAC—eXtensible Randomized Matrix Arithmetic Coding [7] has been used to secure data communication between source nodes to the destination nodes in the particular cluster.

\section{Review of Literature}

A number of methods utilizing sink mobility for data compilation in Wireless Sensor Networks have been proposed in current years [8]. Design of a disseminated energy-efficient clustering algorithm for mixed wireless sensor networks for Cluster-heads [9] are designated by a vision based on the ratio trapped between residual energy of every node and the regular energy of the network [10]. The clustering Algorithm is a kind of key technique used to reduce energy consumption [11]. It can increase the scalability and lifetime of the network. An energy-efficient hybrid data gathering protocol based on the dynamic switching of reporting schemes in wireless sensor networks [12] can be used Practical Expected Delay (PED) \& Particle Swarm Optimization (PSO) algorithm and applicable for both time-driven \& event-driven data reporting process [13]. In Parameter-based Area Detection (PAD) algorithm, it considers only closeness to target area, defined by no. of hops, which include irrelevant sensor nodes [14].

An energy-efficient co-ordination Algorithm for topology in Ad-hoc wireless networks uses sleep and active concept and preserves network connectivity, capacity, decreases latency \& provides significant energy savings [15]. PEAS use probing environment and adaptive sleeping. Achieves robustness and adaptive from minimized protocol operations, reduced message exchanges \& elimination of per-neighboring states. Adaptive Self-Configuring Sensor Networks (ASCENT) technique proposed to reduce the message loss and enlarge in energy efficiency. The low power cost will allow these wireless networks of sensors and actuators to be densely distributed. The nodes in these dense networks will coordinate to perform the distributed sensing tasks [16]. Moreover, as described in this paper, the nodes can also coordinate to make the most of the idleness provided by high density, so as to extend overall system lifetime. It is an adaptive self-configuration topology mechanism for disseminated wireless sensor networks. LEACH reduces frequent energy to the extent that $9 \times$ evaluated with straight broadcast $\& 1^{\text {st }}$ node bereavement in leach take places over 9 times later than $1^{\text {st }}$ node in direct-transmission [17].

In Power-Efficient Gathering in Sensor Information Systems (PEGASIS), all nodes converse only with the neighboring neighbor by regulating its authority signal to be only heard by this contiguous neighbor. Each node uses indication force to determine the distance. A leader is elected from the chain shaped on the basis of residual power of the nodes, in each round and this leader broadcasts the composed data from its contiguous nodes to the Base Station. This approach reduces the overload and lesser the bandwidth prerequisite for the message [18].

In I-LEACH, first of all the probability based selection criteria for a $\mathrm{CH}$ was replaced with concept of residual energy. The other limitation has been resolved out by construction of clusters on the foundation of x-axis coordinates of the nodes; it facilitated out in the standardized allotment of the Cluster Heads [19].

\section{Sleep Active Cluster Routing Protocol}

In the proposed protocol, MSs are mounted upon public buses circulating within urban environments on fixed trajectories and near-periodic schedule. Namely, sinks motion is not controllable and their routes do not adapt 
upon specific WSN deployments. Our only assumption is that sensors are deployed in urban areas in proximity to public transportation vehicle routes. Also, an adequate number of nodes are enrolled as RNs as a fair compromise between a small numbers which results in their rapid energy depletion and a large number which results in reduced data throughput. Finally, SNs are grouped in separate clusters. Raw sensory data are filtered within individual clusters exploiting their inherent spatial-temporal redundancy. Thus, the overhead of multi-hop data relaying (inter-clustering traffic) to the edge RNs is minimized (see Figure 1).

\subsection{Sleep and Active Scheduling}

Wireless sensor networks (WSNs) are self-organized network that consists of a large number of sensor nodes randomly deployed in an area of interest to monitor diverse phenomena. The data collected by these sensors are transferred directly or via a multi-hop routing to a sink node or base station. However, most of these devices are equipped with limited energy supplies, and it is inconvenient to recharge the batteries because nodes may be deployed in a hostile environment. Therefore, it has become one of the crucial problems how to optimize the topology of network and minimize energy consumption to prolong the network lifetime. Hence, the lifetime of a sensor network can be extended by using several energy conservation techniques. Topology control is the primary technique of energy saving, and energy saving is also one of the most important objectives in topology control.

During sleep periods, the radios are completely turned off, and during active periods, they are turned back on to transmit and receive messages. Although the synchronized low duty cycle operation of a sensor network is energy efficient, it has one major deficiency: it increases the packet delivery latency. At a source node, a sampling reading may occur during the sleep period and has to be queued until the active period. An intermediate node may have to wait until the receiver wakes up before it can forward a packet received from its previous hop. This is called sleep latency [12], and if all nodes are synchronized to the same schedule, it increases proportionally with hop length by a slope of schedule length (active period plus sleep period). In scenarios where minimizing sleep latency is not important (non time critical applications), [15] also present an excellent analysis on bounds on the delay of sending data from a node to a sink using a completely decentralized duty cycling scheme. They show that if each sensor turns on and off independent of the other sensors, the delay incurred is proportional to the distance of the node from the sink. However the rate of this linear increase is not dependent on the locations of the nodes, but on the node density, transmission range and the average active and sleep durations.

\subsection{Clustering and Cluster-Head Election}

Clustering is proven to be an effective approach for data aggregation, balancing the load and protracted network

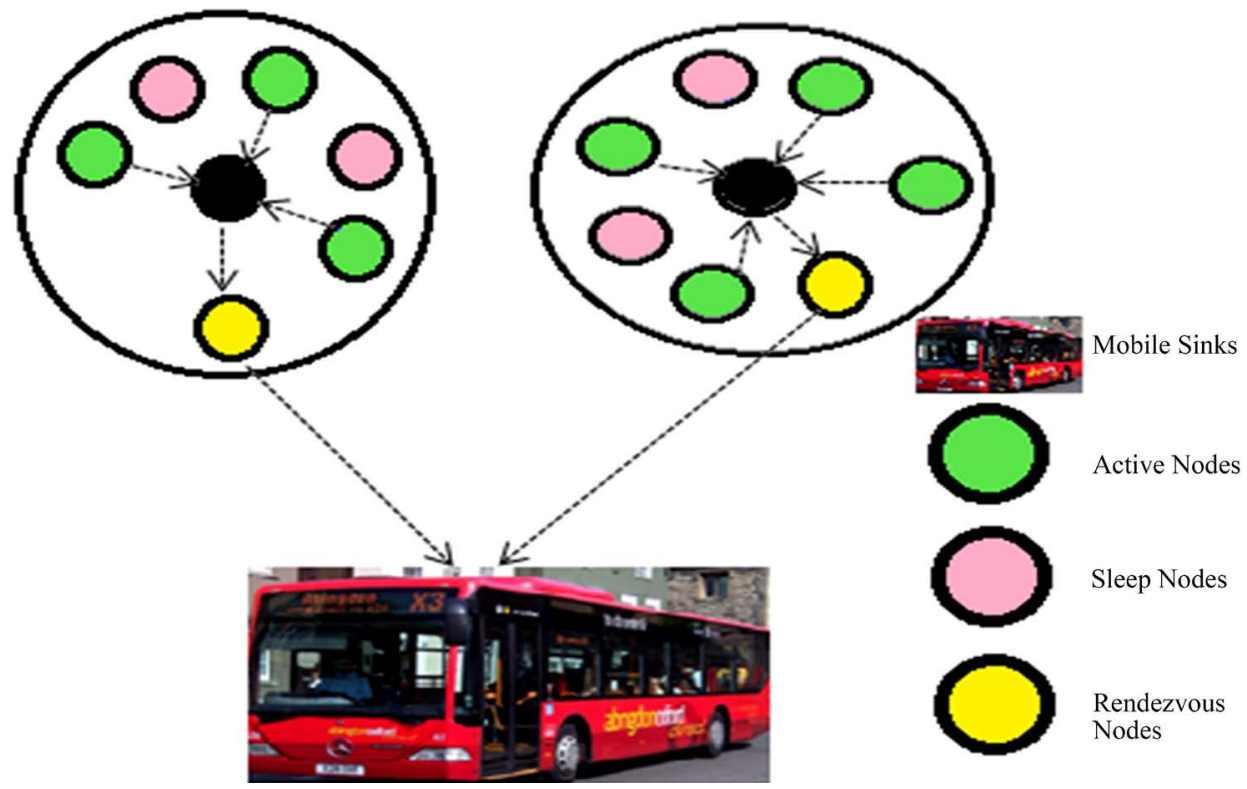

Figure 1. Tryst sensor nodes, cluster structures, and data forwarding paths in mobile-cluster. 
duration. Moreover these, clustering too reduce channel disputation and package crashes, which in turn increases network throughput under elevated load. Universal-LEACH (U-LEACH) is used for constructing the clustering in this approach.

The node that is having relatively high energy will be elected as a Cluster-Head and is changed often so as to provide balanced energy consumption in the midst of the cluster. The elected cluster head will be the active node (Figure 2).

The Universal-Low Energy Adaptive Cluster Hierarchy (U-LEACH) is an energy efficient protocol presentation a noteworthy decrease in the energy expenditure by the sensor nodes. Unlike Low Energy Adaptive Cluster Hierarchy (LEACH), in U-LEACH, the assortment of Cluster-Head depends on the early and the residual energy of the nodes. In a particular cluster, the relocate of in sequence stuck between the nodes takes position by forming a procession, starting from the uttermost node from the base station.

\subsection{Tryst Node Election}

RNs lie within the range of MS's trajectory and suitable RNs are those that are in reporting of all the nodes in the cluster. We set up a RN in each and every cluster, so as to pass the data to the mobile sink from the $\mathrm{CH}$. Each and every cluster has a RN, so as to evade competency between the RNs.

Communication have to start when the connection is available and stop when the connection no longer exists, so that the RN does not carry on to broadcast data when the MS is no longer unloading it. To address this concern, we use an acknowledgment-based protocol stuck between RN and MS. The MS, in all subsequent path traversals after the setup phase, intermittently broadcasts a POLL packet, announcing its presence and soliciting data as it proceeds along the path. The POLL is transmitted at fixed intervals TPOLL (Figure 3).

This POLL packet is used by RNs to notice when the MS is within connectivity diversity. The RN response the POLL will start broadcasting data packets to the MS. The MS acknowledges each received data packet to the RN so that the RN realizes that the connection is active and the data were reliably delivered. The documented information packet can then be vacant from the RN's accumulation. It should be accentuated the conscription of explicit nodes as RNs is subject to change during the steady phase. Thus, if the energy supply of a RN falls below a threshold, it may request the local $\mathrm{CH}$ to engage another node as RN so as to further extend the network's

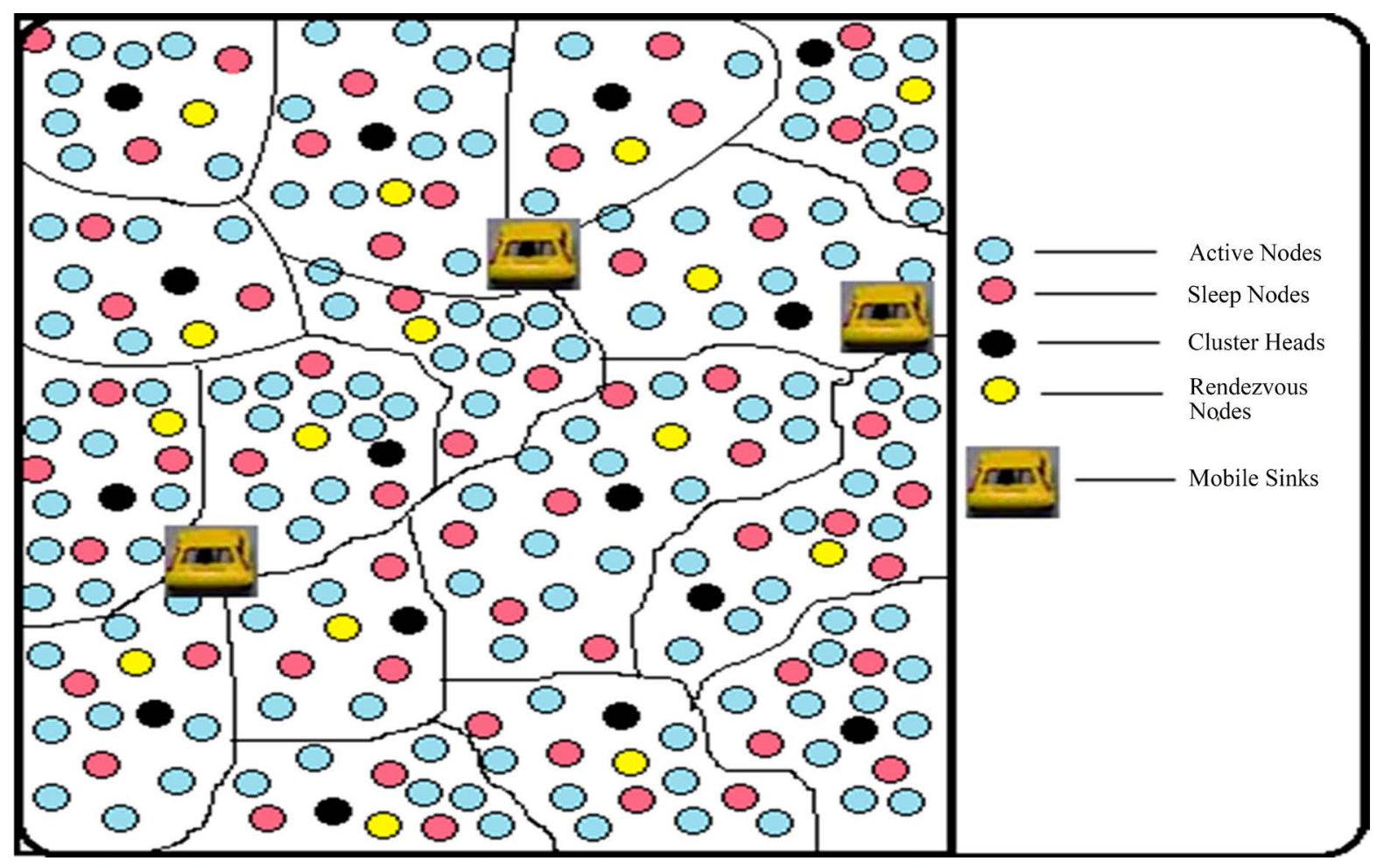

Figure 2. Overall depiction of the network. 


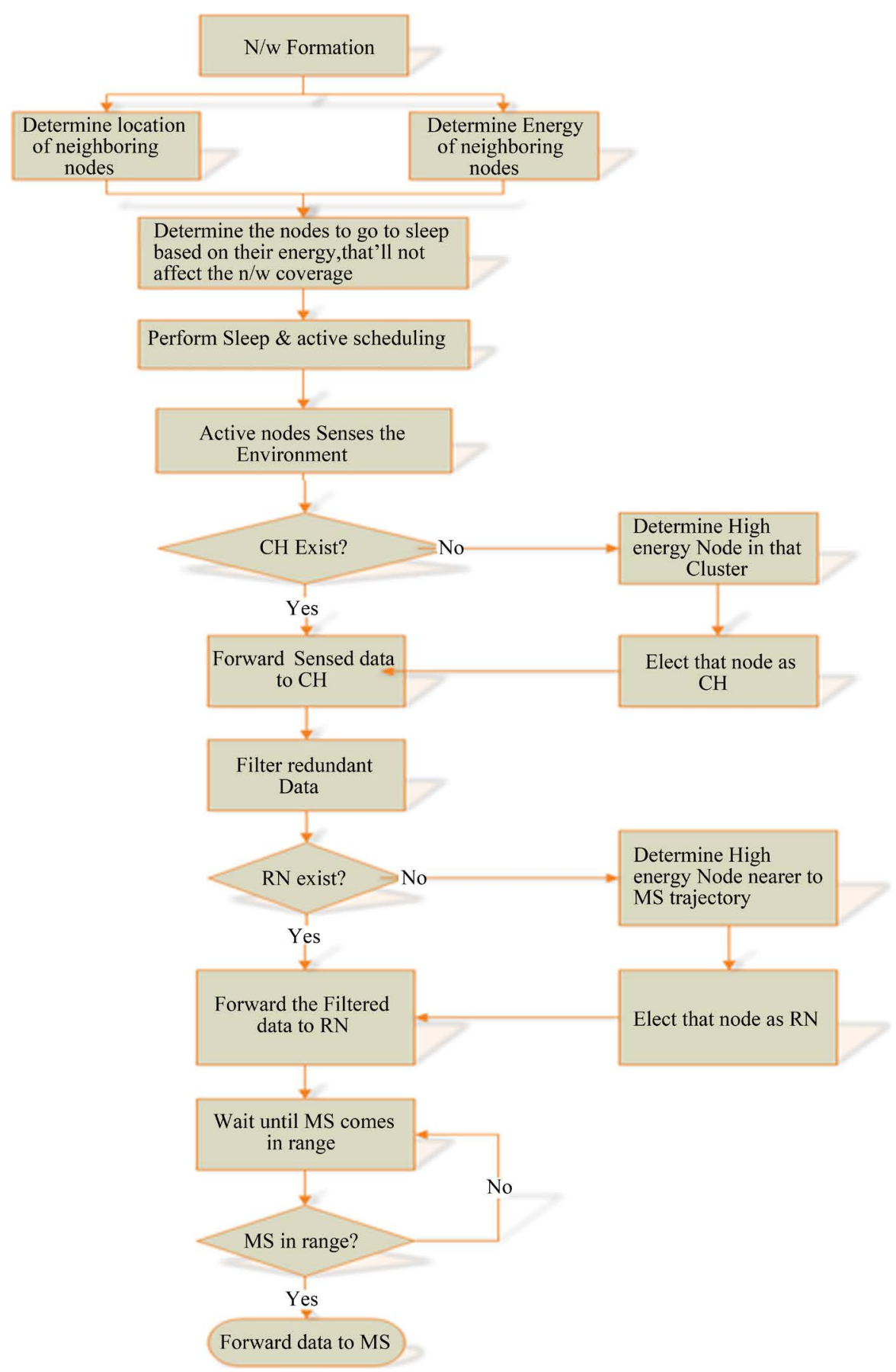

Figure 3. Overall flow of the network.

lifetime without affecting the current clustered infrastructure. To enable RNs substitution, the $\mathrm{CH}$ polls the candidate RNs of the setup phase (excluding the retiring $\mathrm{RN}$ ) to be informed about their current residual energy status and then selects the new RN.

During a training period, all the WSN edge nodes located within the range of MS routes are selected as RNs and construct paths between them with the residue of sensor nodes. Those paths are used by remote nodes to forward their sensory data to RNs; the latter buffer sensory data and deliver them to the MS when it re approaches in range. The movement of mobile robots is controllable which is impractical in realistic urban traffic conditions. 


\subsection{Data Aggregation and Forwarding to RNS}

Data from various SNs sent to their CHs. CHs remove data redundancy and forward it to the RN. The RN receives the data and demands the Mobile Sink to collect the data from it. Each and every cluster has a CH and RN. A MS will collect data from each and every cluster.

The steady phase of Mobile-Cluster protocol starts with the periodic recording of environmental data from sensor nodes with a $\mathrm{T}_{\mathrm{r}}$ period. The data accumulated at individual source nodes are sent to local CHs. CHs perform data processing to remove spatial-temporal data redundancy, which is likely to exist since cluster members are located maximum two hops away.

$\mathrm{CHs}$ then forward filtered data toward remote $\mathrm{CH}$ they are attached to. Alongside the inter-cluster path, a second-level of data filtering may apply. The data sensed by the active sensor nodes and are sent to their corresponding cluster heads, where filtering process takes place. (i.e.) the redundant data removes here, which also helps in reduced energy consumption.

Then, the data from the cluster heads sent to the tryst nodes, located in its cluster. This tryst node is responsible to transmit the data to the mobile sink, when the MS comes in range to it. The RN is not only responsible for transmitting data to the MS, but also responsible for the "sleep and active scheduling”. And, if the CH has been lost due to natural disasters like erosion, high temperature, storm, etc., then that cluster needs a CH immediately, in that case the RN acts as $\mathrm{CH}$ till getting a new $\mathrm{CH}$ in that cluster.

\subsection{XRMAC—eXtensible Randomized Matrix Arithmetic Coding}

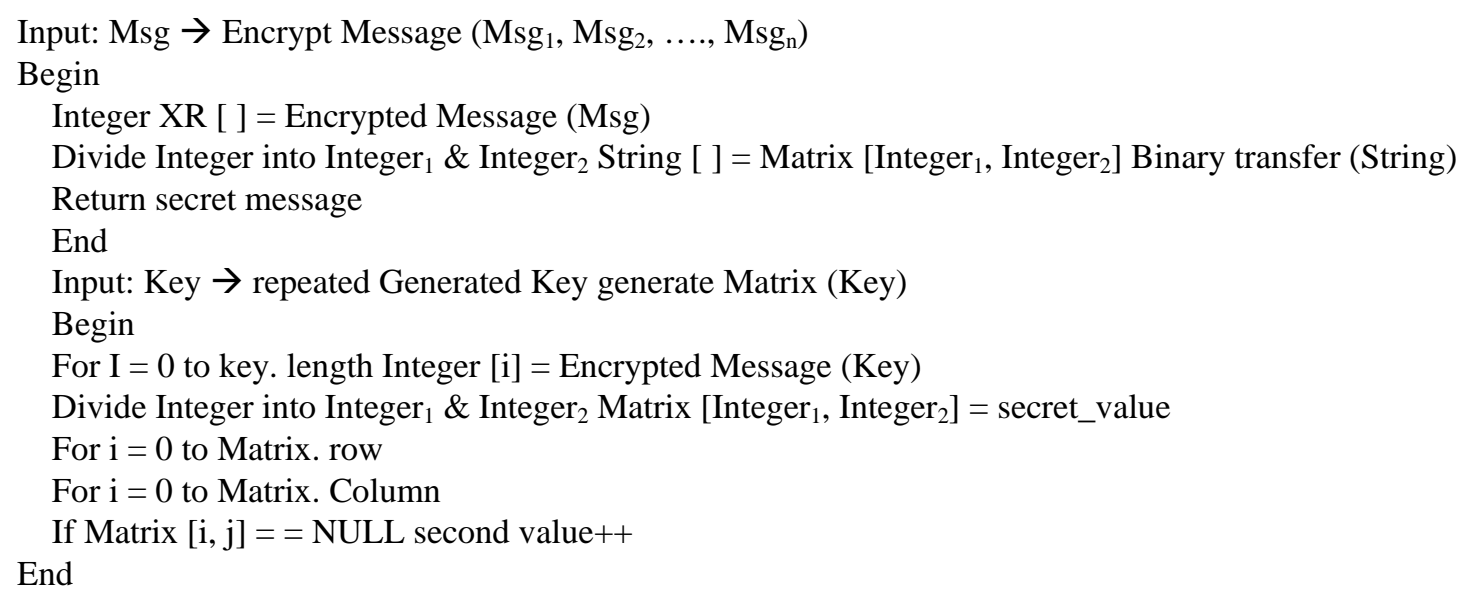

\section{Performance Evaluation}

The SACR protocol has been implemented in Network Simulator-2.34(NS-2.34).

As discussed in the Section 2, a number of tryst-based approaches have been proposed which either assume a fixed MS trajectory or determine that trajectory according to some energy-related optimization criteria (Table 1).

As Mobile-Cluster assume that MS moves on a fixed trajectory, a fair comparison of this protocol with other proposals should only consider the efficiency of routing structures for transferring data from SNs to RNs. In the simulation tests, we compare our SACR protocol result with Mobile Cluster (MOBCL) [8], Geographic Adaptive Fidelity (GAF) [5], U-LEACH [12].

Table 1. Simulation parameters.

\begin{tabular}{cc}
\hline Parameter & Value \\
\hline Number of nodes & $100,200,300,400,500$ \\
Network size & $800 \mathrm{~m} \times 800 \mathrm{~m}$ \\
Node Distribution & Randomly Distributed \\
Data Packet & 4000 bits \\
Environment & Network simulator-2.34(NS-2.34) \\
\hline
\end{tabular}


Figure 4 shows the increased network lifetime of SACR than the others MOBCL, GAF, U-LEACH. Table 2 given below shows the performance variance that describes the graph.

Table 2 explains that lifetime variance of different protocols when the no. of nodes varies. Our proposed protocol shows the increased network lifetime, when comparing with the others.

Figure 5 shows the increased outage of SACR than MOBCL, GAF, U-LEACH protocols.

Table 3 explains that outage variance of different protocols when the no. of nodes varies. Our proposed protocol shows high outage, when comparing with the other protocols.

Figure 6 shows the increased network lifetime of SACR than MOBCL, GAF, U-LEACH. Table 2 given below shows the performance variance that describes the graph.

Figure 7 shows number of packets sensed versus residual energy for the 4 methods and Figure 8 shows the total outage of SACR than MOBCL, GAF, U-LEACH. Table 3 shows the performance variance that describes the graph.

\section{Conclusion}

Here, balanced energy consumption protocol in wireless sensor networks has been designed. Sleep and active

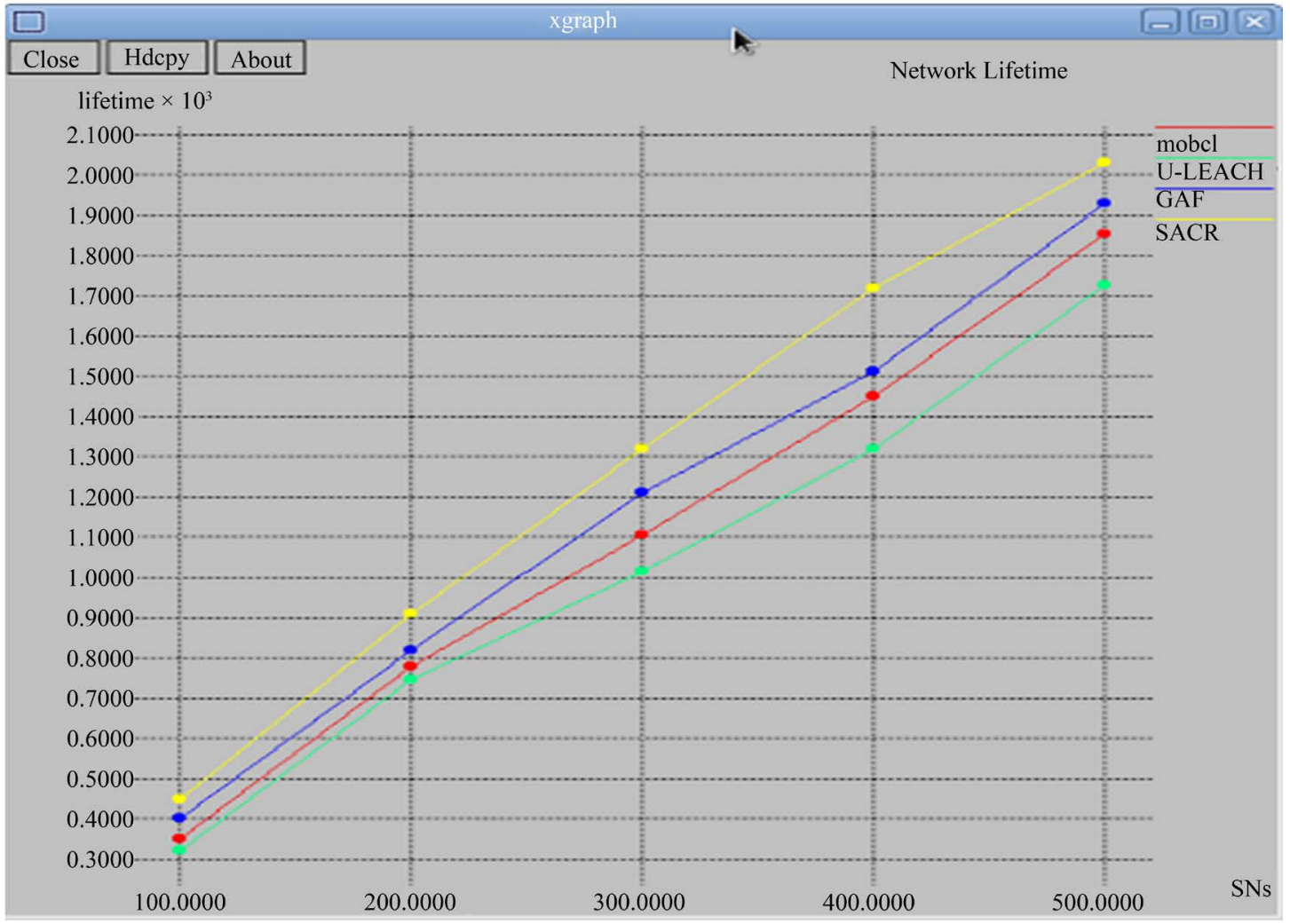

Figure 4. SNs vs. N/W lifetime.

Table 2. No of nodes vs lifetime.

\begin{tabular}{|c|c|c|c|c|}
\hline \multirow{2}{*}{ No of nodes } & \multicolumn{4}{|c|}{ Network Lifetime (sec) } \\
\hline & MOBCL & GAF & U-LEACH & SACR \\
\hline 100 & 350 & 400 & 320 & 450 \\
\hline 200 & 780 & 820 & 745 & 910 \\
\hline 300 & 1105 & 1210 & 1015 & 1320 \\
\hline 400 & 1450 & 1510 & 1320 & 1720 \\
\hline 500 & 1853 & 1930 & 1725 & 2032 \\
\hline
\end{tabular}




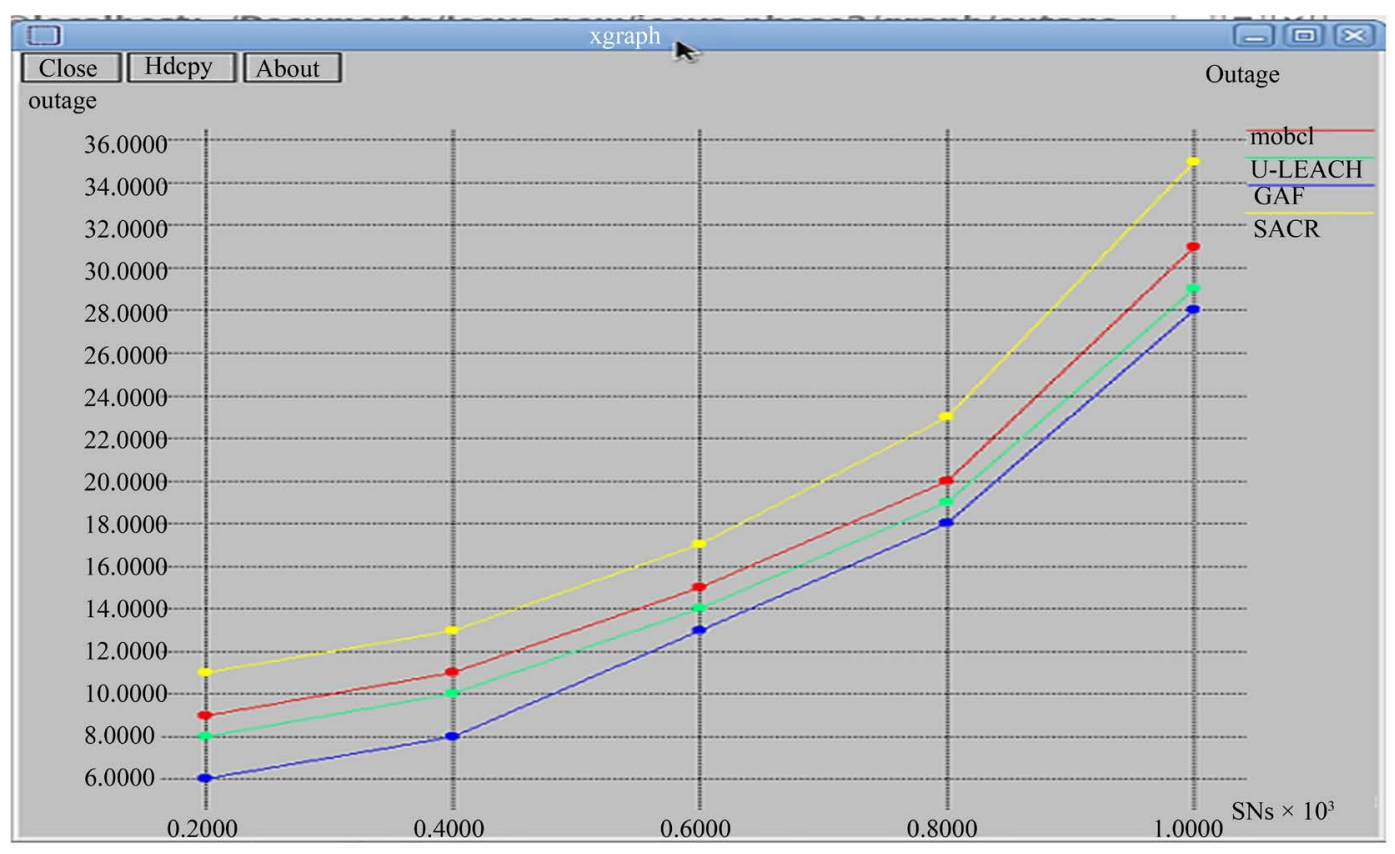

Figure 5. SNs vs. total outage.

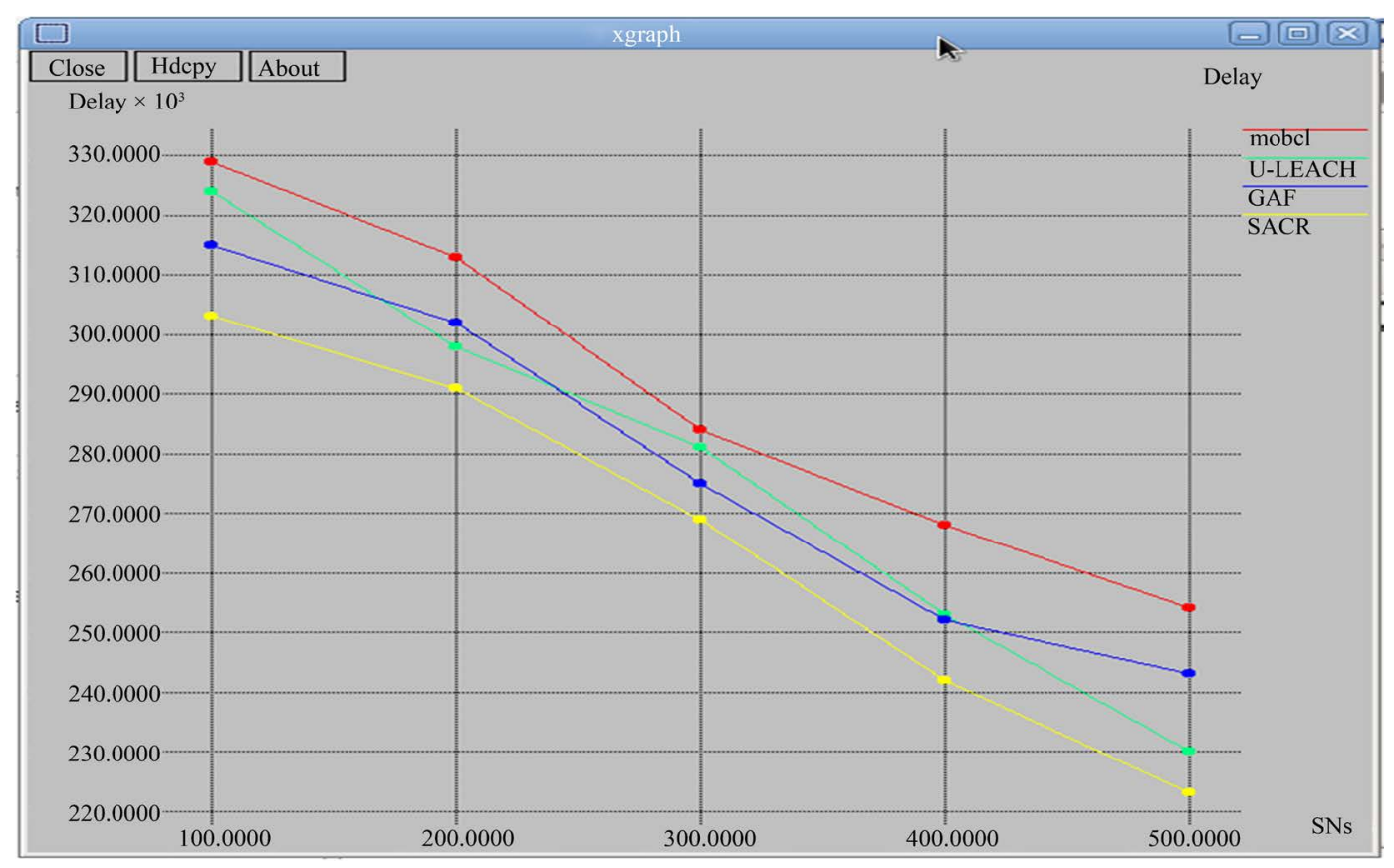

Figure 6. SNs vs. N/W lifetime.

Table 3. No of nodes vs outage.

\begin{tabular}{ccccc}
\hline \multirow{2}{*}{ No of nodes } & \multicolumn{4}{c}{ Network Lifetime (sec) } \\
\cline { 2 - 5 } & MOBCL & GAF & U-LEACH & SACR \\
\hline 100 & 9 & 6 & 8 & 11 \\
200 & 11 & 8 & 10 & 13 \\
300 & 15 & 13 & 14 & 17 \\
400 & 20 & 18 & 23 & 35 \\
500 & 31 & 28 & 29 & 35 \\
\hline
\end{tabular}




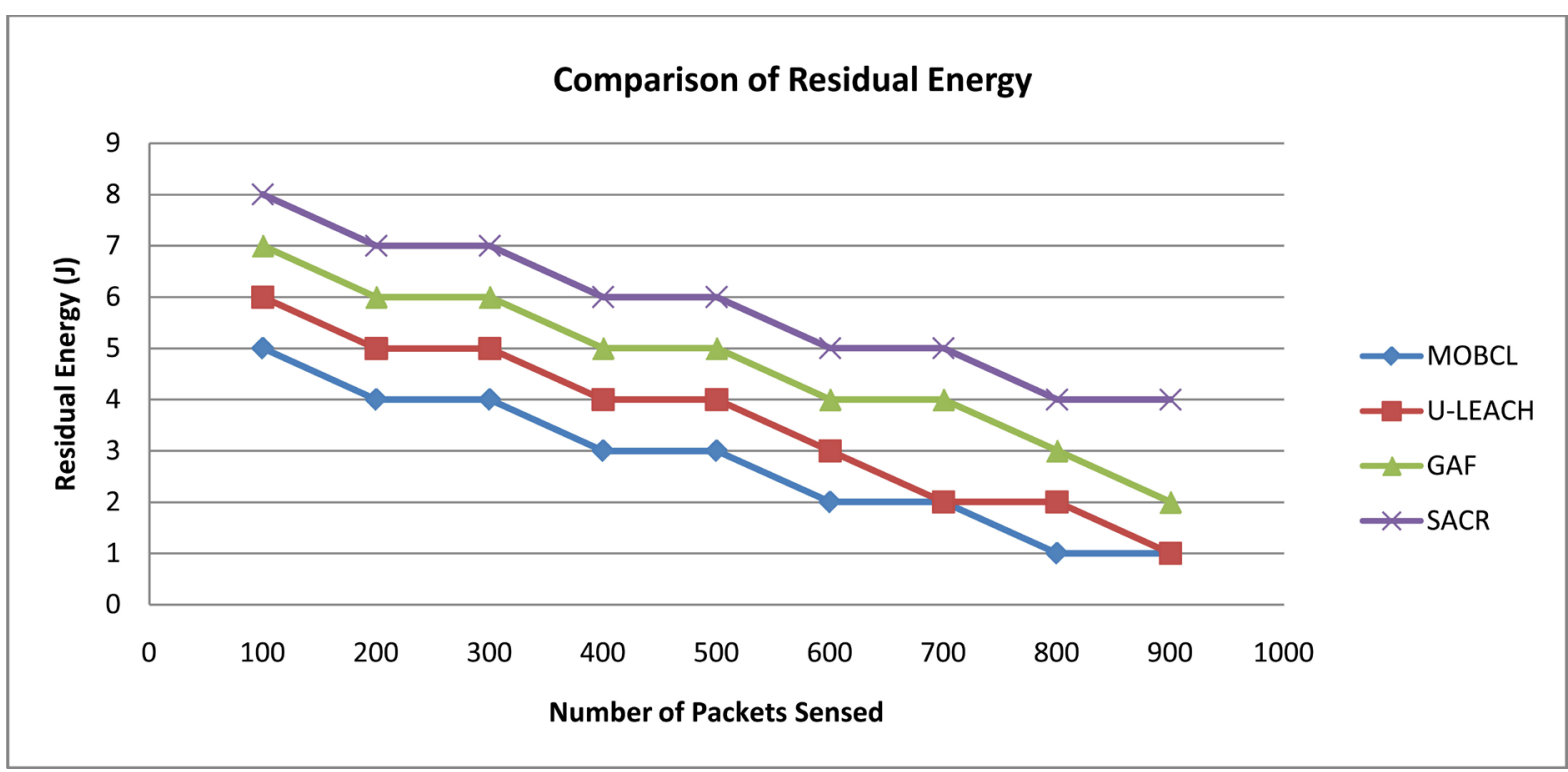

Figure 7. Number of packets sensed vs residual energy (J).

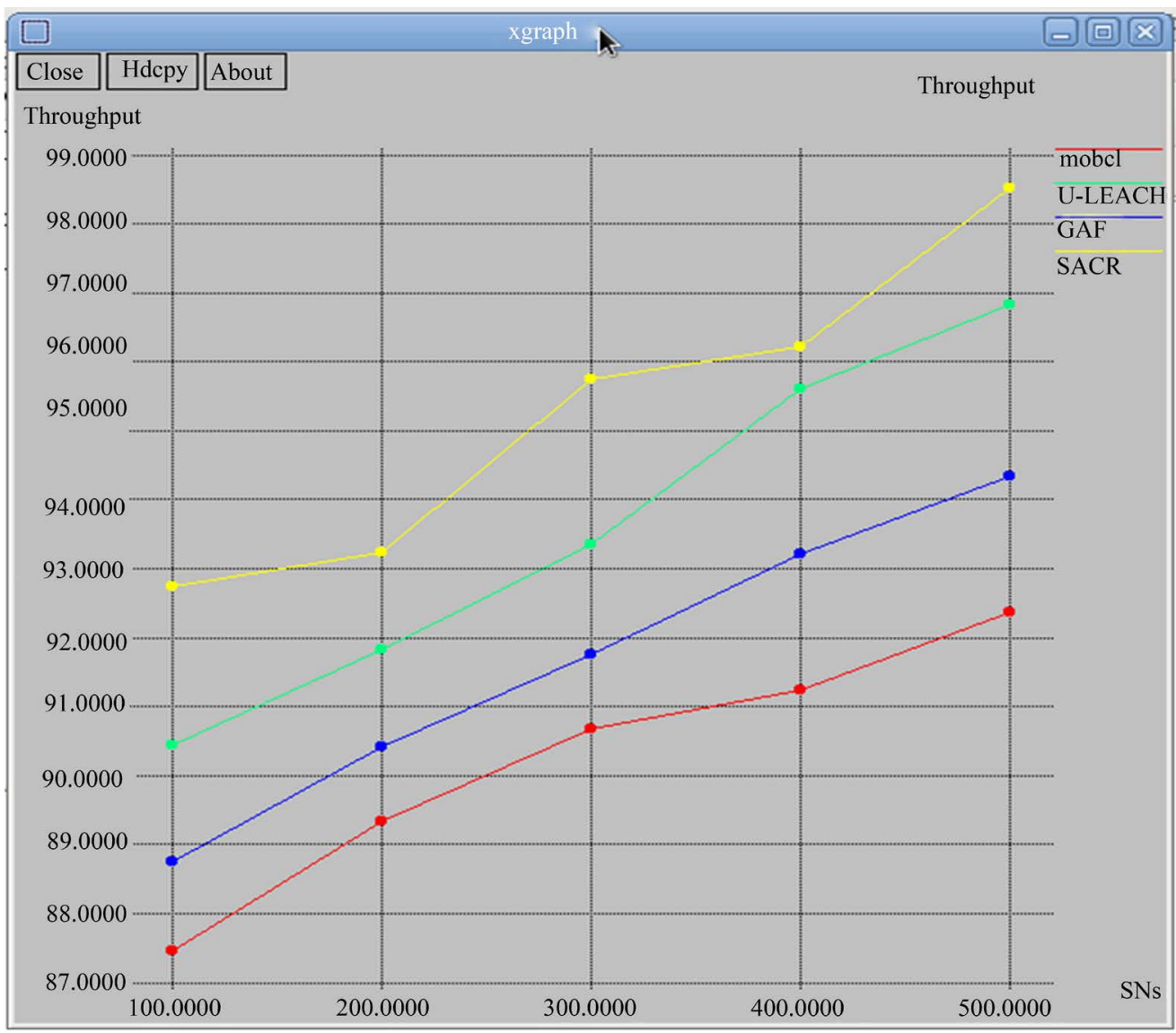

Figure 8. SNs vs. total outage.

scheduling is used to reduce the energy consumption by taking the nodes to the sleep and active state periodically. Then, cluster head has been used to reduce the transmission of redundant data by data aggregation. Tryst node helps in data transfer to the mobile sinks, when it comes in range. It also organizes the cluster head election 
and sleep and active scheduling. The mobile sinks also help in reduced energy consumption, by collecting the data in each and every cluster itself. Finally, these cluster heads, tryst nodes, sleep and active scheduling combine helps in balanced energy consumption, which in turn increases the network lifetime obviously from the Simulation Results. Wireless sensor network lifetime improvement is very important issue in wireless sensor network.

\section{References}

[1] Zebbane, B., Chenait, M. and Badache, N. (2012) Towards an Energy-Efficient Algorithm based Sleep-Scheduling for Wireless Sensor Networks. 5th International Conference on New Technologies, Mobility and Security (NTMS), Istanbul, 7-10 May 2012, 1-4. http://dx.doi.org/10.1109/ntms.2012.6208725

[2] Chen, B., Jamieson, K., Balakrishnan, H. and Morris, R. (2002) Span: An Energy-Efficient Coordination Algorithm for Topology Maintenance in Ad Hoc Wireless Networks. Wireless Networks, 8, 481-494.

[3] Estrin, D., Govindan, R., Heidemann, J. and Kumar, S. (1999) Next Century Challenges: Scalable Coordination in Sensor Networks. Proceedings of the 5th Annual ACM/IEEE International Conference on Mobile Computing and Networking, 263-270.

[4] Harold Robinson, Y., Rajaram, M., Golden Julie, E. and Balaji, S. (2016) Dominating Set Algorithm and Trust Evaluation Scheme for Secured Cluster Formation and Data Transferring. World Academy of Science, Engineering and Technology, International Journal of Computer, Electrical, Automation, Control and Information Engineering, 10, $388-393$.

[5] Olariu, S. and Stojmenović, I. (2006) Design Guidelines for Maximizing Lifetime and Avoiding Energy Holes in Sensor Networks with Uniform Distribution and Uniform Reporting. INFOCOM, 1-12

[6] Manikandan, K. and Purushothaman, T. (2010) An Efficient Routing Protocol for Distributed Wireless Sensor Networks. International Journal of Computer Applications, 10, 73-86.

[7] Rajaram, M., Balaji, S. and Jeeva, R. (2013) XRMAC-An Extended RMAC Scheme to Evade Hacking by Dynamic Sizing. 2013 Fifth International Conference on Advanced Computing (ICoAC), 158-163.

[8] Robinson, Y.H. and Rajeswari, S.R. (2011) Energy-Based Dynamic Encryption for Wireless Sensor Networks. Wireless Communication, 3, 661-663.

[9] Harold Robinson, Y. and Rajaram, M. (2014) A Novel Approach to Allocate Channels Dynamically in Wireless Mesh Networks. World Academy of Science, Engineering and Technology, International Journal of Computer, Electrical, Automation, Control and Information Engineering, 8, 1865-1868.

[10] Harold Robinson, Y. and Rajaram, M. (2015) Trustworthy Link Failure Recovery Algorithm for Highly Dynamic Mobile Adhoc Networks. World Academy of Science, Engineering and Technology, International Journal of Electrical, Computer, Energetic, Electronic and Communication Engineering, 9, 233-236.

[11] Lindsey, S. and Raghavendra, C.S. (2002) PEGASIS: Power Efficient Gathering in Sensor Information Systems. IEEE Aerospace Conference Proceedings, Vol. 3, 3-1125.

[12] Kang, S.H. and Nguyen, T. (2012) Distance Based Thresholds for Cluster Head Selection in Wireless Sensor Networks. IEEE Communications Letters, 16, 1396-1399.

[13] Golden Julie, E., Tamil Selvi, S. and Harold Robinson, Y. (2014) Opportunistic Routing with Secure Coded Wireless Multicast Using MAS Approach. World Academy of Science, Engineering and Technology, International Journal of Computer, Electrical, Automation, Control and Information Engineering, 8, 1247-1250.

[14] Balaji, S. and Rajaram, M. (2014) EUDIS-An Encryption Scheme for User-Data Security in Public Networks. International Journal of Computer, Electrical, Automation, Control and Information Engineering, World Academy of Science, Engineering and Technology, 95, 2029-2034.

[15] Harold Robinson, Y. and Rajaram, M. (2016) A Memory Aided Broadcast Mechanism with Fuzzy Classification on a Device-to-Device Mobile Ad Hoc Network. Wireless Personal Communications, 1-23. http://dx.doi.org/10.1007/s11277-016-3213-0

[16] Harold Robinson, Y. and Rajaram, M. (2015) Energy-Aware Multipath Routing Scheme Based on Particle Swarm Optimization in Mobile Ad Hoc Networks. The Scientific World Journal, 1-9. http://dx.doi.org/10.1155/2015/284276

[17] Harold Robinson, Y. and Rajaram, M. (2015) Establishing Pairwise Keys Using Key Predistribution Schemes for Sensor Networks. World Academy of Science, Engineering and Technology International Journal of Computer, Electrical, Automation, Control and Information Engineering, 9, 608-612.

[18] Ramalakshmi, S. and Robinson, Y.H. (2014) ATMPH: Attaining Optimal Throughput Capacity of MANET with Power Control in Heterogeneous Network. Programmable Device Circuits and Systems, 6, 111-115.

[19] Balaji, S. and Rajaram, M. (2016) SIPTAN: Securing Inimitable and Plundering Track for Ad Hoc Network. Wireless Personal Communications, Springer, US, 1-21. http://dx.doi.org/10.1007/s11277-016-3187-y 


\section{Submit or recommend next manuscript to SCIRP and we will provide best service for you:}

Accepting pre-submission inquiries through Email, Facebook, Linkedin, Twitter, etc A wide selection of journals (inclusive of 9 subjects, more than 200 journals)

Providing a 24-hour high-quality service

User-friendly online submission system

Fair and swift peer-review system

Efficient typesetting and proofreading procedure

Display of the result of downloads and visits, as well as the number of cited articles

Maximum dissemination of your research work

Submit your manuscript at: http://papersubmission.scirp.org/ 